\title{
Genetic and morphological analyses indicate that the Australian endemic scorpion Urodacus yaschenkoi (Scorpiones: Urodacidae) is a species complex
}

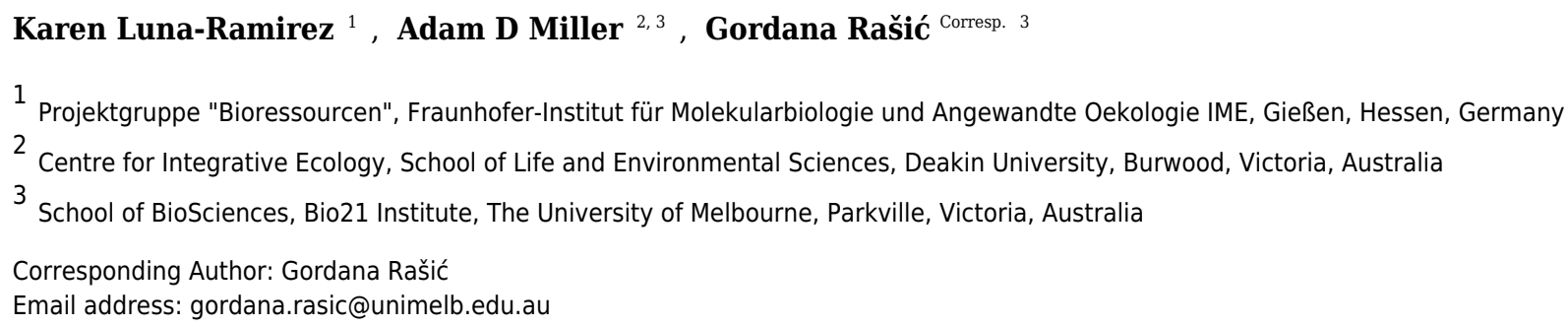

Background. Australian scorpions have received far less attention from researchers than their overseas counterparts. Here we provide the first insight into the molecular variation and evolutionary history of the endemic Australian scorpion Urodacus yaschenkoi. Also known as the inland robust scorpion, it is widely distributed throughout arid zones of the continent and is emerging as a model organism in biomedical research due to the chemical nature of its venom. Methods. We employed Bayesian Inference (BI) methods for the phylogenetic reconstructions and divergence dating among lineages, using unique haplotype sequences from two mitochondrial loci (COXI, 16S) and one nuclear locus (28S). We also implemented two DNA taxonomy approaches (GMYC and PTP/dPTP) to evaluate the presence of cryptic species. Linear Discriminant Analysis was used to test whether the linear combination of 21 variables (ratios of morphological measurements) can predict individual's membership to a putative species. Results. Genetic and morphological data suggest that $U$. yaschenkoi is a species complex. High statistical support for the monophyly of several divergent lineages was found both at the mitochondrial loci and at a nuclear locus. The extent of mitochondrial divergence between these lineages exceeds estimates of interspecific divergence reported for other scorpion groups. The GMYC model and the PTP/bPTP approach identified major lineages and several sub-lineages as putative species. Ratios of several traits that approximate body shape had a strong predictive power (83-100\%) in discriminating two major molecular lineages. A time-calibrated phylogeny dates the early divergence at the onset of continental-wide aridification in late Miocene and Pliocene, with finer-scale phylogeographic patterns emerging during the Pleistocene. This structuring dynamics is congruent with the diversification history of other fauna of the Australian arid zones. Discussion. Our results indicate that the taxonomic status of $U$. yaschenkoi requires revision, and we provide recommendations for such future 
efforts. A complex evolutionary history and extensive diversity highlights the importance of conserving $U$. yaschenkoi populations from different Australian arid zones in order to preserve patterns of endemism and evolutionary potential. 
1 Title: Genetic and morphological analyses indicate that the Australian

2 endemic scorpion Urodacus yaschenkoi (Scorpiones: Urodacidae) is a species

3 complex

4

5 Authors: Luna-Ramírez $\mathrm{K}^{\mathrm{a}}$, Miller $\mathrm{AD}^{\mathrm{b}, \mathrm{c}}$, Rašić $\mathrm{G}^{\mathrm{b}^{*}}$

6 a Museum Victoria, 11 Nicholson St., Carlton Gardens, Melbourne, VIC 3053, Australia.

7 (Current affiliation: Fraunhofer-Institut für Molekularbiologie und Angewandte Oekologie IME,

8 Projektgruppe "Bioressourcen" Heinrich-Buff-Ring 58/62, Gießen 35392; Germany).

9 bPest and Environmental Adaptation Research Group, School of BioSciences, The University of

10 Melbourne, Victoria 3010, Australia.

$11{ }^{c}$ Centre for Integrative Ecology, School of Life and Environmental Sciences, Deakin University,

12 Victoria 3280, Australia.

$14{ }^{*}$ Corresponding author: Gordana Rašić, Pest and Disease Vector Group, School of BioSciences,

15 The University of Melbourne, Victoria 3010, Australia;

16 phone number: +6139035 5237, fax number: +6138344 2279, Email:

17 rasic.gordana@gmail.com 
19 Running title: Scorpion Urodacus yaschenkoi is a species complex 


\section{Abstract}

22 Background. Australian scorpions have received far less attention from researchers than their

23 overseas counterparts. Here we provide the first insight into the molecular variation and

24 evolutionary history of the endemic Australian scorpion Urodacus yaschenkoi. Also known as

25 the inland robust scorpion, it is widely distributed throughout arid zones of the continent and is

26 emerging as a model organism in biomedical research due to the chemical nature of its venom.

27 Methods. We employed Bayesian Inference (BI) methods for the phylogenetic reconstructions

28 and divergence dating among lineages, using unique haplotype sequences from two

29 mitochondrial loci $(C O X I, 16 S)$ and one nuclear locus $(28 S)$. We also implemented two DNA

30 taxonomy approaches (GMYC and PTP/dPTP) to evaluate the presence of cryptic species.

31 Linear Discriminant Analysis was used to test whether the linear combination of 21 variables

32 (ratios of morphological measurements) can predict individual's membership to a putative

33 species.

34 Results. Genetic and morphological data suggest that $U$. yaschenkoi is a species complex. High

35 statistical support for the monophyly of several divergent lineages was found both at the

36 mitochondrial loci and at a nuclear locus. The extent of mitochondrial divergence between these

37 lineages exceeds estimates of interspecific divergence reported for other scorpion groups. The

38 GMYC model and the PTP/bPTP approach identified major lineages and several sub-lineages as

39 putative species. Ratios of several traits that approximate body shape had a strong predictive

40 power (83-100\%) in discriminating two major molecular lineages. A time-calibrated phylogeny

41 dates the early divergence at the onset of continental-wide aridification in late Miocene and

42 Pliocene, with finer-scale phylogeographic patterns emerging during the Pleistocene. This 
43 structuring dynamics is congruent with the diversification history of other fauna of the Australian

44 arid zones.

45 Discussion. Our results indicate that the taxonomic status of $U$. yaschenkoi requires revision, and

46 we provide recommendations for such future efforts. A complex evolutionary history and

47 extensive diversity highlights the importance of conserving $U$. yaschenkoi populations from

48 different Australian arid zones in order to preserve patterns of endemism and evolutionary

49 potential. 


\section{Introduction}

52

53 Scorpions represent an ancient arthropod lineage that first appeared in the Silurian, and fossil 54 records indicate their bodyplan remained largely unchanged since the Paleozoic period (Dunlop 55 2010; Jeram 1997; Kjellesvig-Waering 1986). Given this relative morphological stasis over long 56 periods of time, the placement of scorpions within Arachnida and internal evolutionary

57 relationships inferred solely from morphological characters have long been contentious (Prendini

58 \& Wheeler 2005; Sharma et al. 2014; Shultz 2007; Soleglad \& Fet 2003). A recent phylogenomic study based on the transcriptome-wide variation suggested non-monophyly of all

60 scorpion superfamilies and several families, largely contradicting the traditional morphology61 based hypotheses (Sharma et al. 2015).

62 The well-supported phylogenetic reconstructions and taxonomy of scorpions are critical for their 63 effective conservation. Scorpion populations can be sensitive to environmental changes due to a 64 low reproductive rate (long generation time, long gestation time, small litter size) and high mortality of immature females (Fet et al. 1998; Lourenço \& Cuellar 1995). Several species have

66 gained threatened status due to over-harvesting for the souvenir and exotic pet trades (CITES,

67 Appendix II, http://www.cites.org/eng/app/appendices.php). Scorpions might also become more

68 harvested for their venom that is increasingly regarded as a source of new therapeutic and

69 insecticidal agents (Gurevitz et al. 2007; Possani et al. 2000; Rodríguez de la Vega et al. 2010).

70 An extensive venom characterization can be found for individual taxa (e.g.(Luna-Ramírez et al.

71 2013; Xu et al. 2014), but a deeper understanding of the evolution of scorpion venoms and their

72 molecular characteristics has been limited by the lack of underlying species tree (Sharma et al. 73 2015). 
74 Extant scorpions inhabit a diversity of terrestrial habitats across all continents except Antarctica,

75 with the greatest species diversity found in tropical and subtropical regions of the world

76 (Lourenço 2001; Prendini 2010). Australian scorpions have received far less attention from

77 researchers than their overseas counterparts. Over 40 scorpion species described in Australia are

78 traditionally organized into four families: Buthidae, Bothriuridae, Urodacidae and Hormuridae

79 (Koch 1977; Monod \& Prendini 2015; Volschenk et al. 2008). The Urodacidae is an Australian

80 endemic family found across the continent, except on the south-eastern seaboard. The family was

81 first described by Koch (1977) that under the current classification includes two genera:

82 Urodacus and the recently described troglobitic Aops (Volschenk \& Prendini 2008). The genus

83 Urodacus contains 20 species described based on morphological characters (Volschenk et al.

84 2012), with many likely undescribed species.

85 Urodacus yaschenkoi (Birula 1903), commonly known as the inland robust scorpion, occupies

86 Australian desert habitats stretching from north-western Victoria through South Australia and

87 across to Western Australia (Walker et al. 2003. )(Fig1). It is emerging as a model organism in

88 toxinology because it produces large volumes of venom compared with other Urodacus species

89 (Luna-Ramírez et al., 2013; Luna-Ramírez et al., 2014). This scorpion has had several synonyms

90 throughout its taxonomic history, starting from the original description as Hemihoplopus

91 yaschenkoi (Birula 1903), followed by Urodacus granifrons (Kraepelin 1916), U. fossor

92 (Kraepelin 1916), and U. kraepelini (Glauert 1963), and finally by U. yaschenkoi (Birula) (Koch

93 1977). Since then, studies of variation in U. yashenkoi populations have not been conducted.

94 Here we provide the first molecular analysis of phylogenetic patterns and history of $U$.

95 yaschenkoi sampled across its native range. DNA sequence data from mitochondrial and nuclear

96 loci, complemented with the analysis of several body-proportion characters, showed that $U$. 
97 yaschenkoi shares a complex diversification history with other Australian arid-adapted fauna.

98 Moreover, the existence of several deeply divergent lineages that also differ in body-shape

99 indicate that further revision of this taxon is warranted.

\section{Materials and Methods}

101

102

\section{Biological material}

103 Samples of Urodacus yaschenkoi were obtained from field and museum collections (Table 1).

104 Live specimens were collected from eight locations (approximately $500 \mathrm{~m}^{2}$ ) in the semi-arid and arid regions of Central Australia in December 2010 and October 2011 (Table 1 and Fig1). Individuals were collected at night from pitfall traps set in front of their burrows, and those outside their burrows were detected using ultraviolet (UV) lamps that reveal soluble fluorescent components ( $\beta$-carboniles) in the scorpion exoskeleton (Stachel et al. 1999) . Captured scorpions were kept alive and transported to the laboratory for morphological identification according to Koch (1977). Key diagnostic feature that distinguishes U. yaschenkoi from other Urodacus

111 species is a very small terminal prolateral tarsus unguis. All specimens were handled according

112 to good animal practices defined by the Government of Australia, and all institutions and

113 museums involved approved the animal handling work. Scorpions were anaesthetized by cooling

114 in a refrigerator $\left(4^{\circ} \mathrm{C}\right)$ for $5 \mathrm{~min}$ before removing $\sim 1 \mathrm{~mm}^{2}$ of leg muscle tissue, which was stored

115 in $90 \%$ ethanol at $4{ }^{\circ} \mathrm{C}$ or $-20^{\circ} \mathrm{C}$ for subsequent DNA extraction. Additional samples were

116 obtained from collections at the South Australian Museum (SAM) and Western Australian

117 Museum (WAM) containing specimens collected between 2000 and 2010 (Table 1). 


\section{DNA extraction, amplification and sequencing}

119 Total DNA was extracted from the stored muscle tissue using the DNeasy Blood and Tissue Kit

120 (Qiagen, Venlo, Netherlands) following the manufacturer's instructions. Two mitochondrial loci

121 (cytochrome oxidase subunit I, COXI; large ribosomal subunit, 16S) and a single nuclear locus

$122(28 S)$ were amplified by PCR with a reaction volume of $20 \mu$ containing $0.5 \mathrm{ng}$ of template

123 DNA, $10 \mu 1$ of Go Taq Master Mix (Promega, Madison, Wisconsin, USA), $0.5 \mu 1$ of $10 \mathrm{nM}$

124 primers and $7 \mu 1$ of RNase-free water (Qiagen). The primer sequences and PCR amplicon sizes

125 are summarized in Table 2.

126 Primers previously designed for the insect COXI gene (Simon et al. 1994; Tanaka et al. 2001)

127 were used to amplify a 630-base pair (bp) fragment from the 3 ' end of the locus. The

128 amplification conditions comprised an initial denaturing step at $95^{\circ} \mathrm{C}$ for $5 \mathrm{~min}$ followed by 35

129 cycles of denaturing at $94^{\circ} \mathrm{C}$ for $30 \mathrm{~s}$, annealing at $52^{\circ} \mathrm{C}$ for $40 \mathrm{~s}$, and extension at $72^{\circ} \mathrm{C}$ for $45 \mathrm{~s}$,

130 and a final extension phase at $72^{\circ} \mathrm{C}$ for $5 \mathrm{~min}$. For the mitochondrial $16 \mathrm{~S}$ gene, the scorpion-

131 specific primer pairs modified by (Gantenbein et al. 2005b) were used to amplify a 425-bp region

132 at the $3^{\prime}$ end of the locus. The amplification conditions comprised an initial denaturing step at

$13394^{\circ} \mathrm{C}$ for $4 \mathrm{~min}$ followed by 30 cycles of denaturing at $94^{\circ} \mathrm{C}$ for $30 \mathrm{~s}$, annealing at $47.5^{\circ} \mathrm{C}$ for 30

$134 \mathrm{~s}$, and extension at $72^{\circ} \mathrm{C}$ for $30 \mathrm{~s}$, and a final extension phase at $72^{\circ} \mathrm{C}$ for $7 \mathrm{~min}$. The $C O X I$ and

$13516 S$ gene fragments were also amplified from three specimens keyed out as Urodacus manicatus

136 (Um2714, Um1814) and U. novaehollandiae (Un2112, Table 1). Sequences from these taxa

137 were used as outgroups in downstream phylogenetic reconstruction. Primer pairs R1S and R1AS,

138 and R2S and R2AS, designed by (Arabi et al. 2012), were used to amplify 1158-bp and 1246-bp

139 fragments of the $28 S$ locus, respectively. Each set of primers amplifies a different region of the

140 gene, which overlaps by $327 \mathrm{bp}$, and their sequences were concatenated to form a larger product 
141 of $2076 \mathrm{bp}$. The amplification conditions for both sets of primers comprised an initial denaturing

142 step at $94^{\circ} \mathrm{C}$ for $4 \mathrm{~min}$, followed by 30 cycles of denaturing at $94^{\circ} \mathrm{C}$ for $30 \mathrm{~s}$, annealing at $55^{\circ} \mathrm{C}$

143 for $30 \mathrm{~s}$, and extension at $72^{\circ} \mathrm{C}$ for $30 \mathrm{~s}$, and a final extension phase at $72^{\circ} \mathrm{C}$ for $7 \mathrm{~min}$.

144 Museum specimens that were not stored under ideal conditions for preservation failed to yield

$145 C O X I$ amplicons suitable for direct sequencing. To address this issue, additional PCR primers

146 were designed to amplify smaller fragments for COXI locus (Table 2), resulting in amplicons of

$147150 \mathrm{bp}$ that were used for subsequent analysis. For the SAM specimens, the amplification of the

$14828 S$ nuclear gene failed entirely and these samples were excluded from further analysis of the

149 nuclear gene variation. All amplicons were sequenced in both directions using the PCR

150 amplification primers, and carried out on an Applied Biosystems 3130 genetic analyzer by

151 Macrogen Inc. (Seoul, South Korea).

152 Sequences were aligned and edited in Geneious Pro v6.1 (Biomatters Ltd) using the MUSCLE

153 alignment option with default parameters. All chromatograms were checked for the presence of

154 multiple peaks (which indicate heterozygosity), and authenticity of the COX1 coding gene was

155 validated by checking for indels and premature stop codons. After this editing process, the

156 alignment of the mitochondrial gene fragments yielded 616-bp and 396-bp products for the

$157 C O X 1$ and $16 S$ genes respectively, and the final $28 S$ alignment was $2076 \mathrm{bp}$ in length. The final

158 dataset contained 68 sequences for each of the mitochondrial genes and 27 sequences for the $28 S$

159 locus (Table 1, [GenBank accession \# KP176717-KP176786]). Shared haplotypes were

160 identified and the uncorrected pairwise genetic distances (\%) were calculated using Geneious Pro

161 v6.1 (Biomatters Ltd). This simple distance measure was implemented to achieve reliable

162 estimates of both intraspecific and interspecific genetic variation. 


\section{Phylogenetic analysis}

164 Phylogenetic reconstructions and divergence dates among lineages were calculated using unique

165 haplotypes and Bayesian Inference (BI) methods implemented in BEAST v2.1.3 (Bouckaert et

166 al. 2014). We used jModeltest v0.1.1 (Posada 2008) to select the best-fit model of evolution,

167 based on Akaike Information Criteria (AIC)(Akaike \& Company 1981) for each of the

168 mitochondrial and nuclear genes (GTR $+\mathrm{G}$ in each case). Mitochondrial loci were combined for

169 analysis due to their similar modes of evolution (GTR+R), as indicated by the incongruence-

170 length difference (ILD) tests (Farris et al. 1995) implemented in PAUP_4.0b10 (Swofford 2002).

171 The nuclear gene $(28 S)$ was analyzed independently due to inconsistencies in taxon sampling

172 (Table 1).

173 Operators were auto-optimized, and five independent Markov Chain Monte Carlo (MCMC) runs

174 were performed using a Yule (speciation) tree-prior, each running for $5 \times 10^{6}$ generations,

175 sampling every 10,000 states. Log files were examined with Tracer v1.5 (Drummond \& Rambaut

176 2007) to ensure that runs were sampling from the same posterior distribution, to determine

177 appropriate burn-in, and to ensure that effective sample sizes (ESSs) of parameters of interest

178 were greater than 1000. Tree files of independent runs were then combined using LogCombiner

179 v2.1.3 (Drummond et al. 2012), discarding the first 20\% and re-sampling at a lower frequency of

180 15,000. The maximum clade credibility (MCC) tree was recovered from a sample of 10,000

181 posterior trees, and branch support was annotated using TreeAnnotator v2.1.3 (Drummond et al.

182 2012). Each analysis started with a random starting tree and seed with no root specified.

183 Sequence data from species of the same genus (U. manicatus and U. novaehollandiae) were used

184 to estimate the root of the mitochondrial gene tree. 
185 Additional phylogenetic constructions were also performed using a truncated $C O X I$ alignment to

186 test the influence of missing data on the final tree topology. Because numerous museum

187 collections yielded short COXI gene products, we trimmed the alignment to 150-bp to exclude

188 regions of the alignment with high levels of missing data. This exercise demonstrated that the

189 inclusion/exclusion of missing data had little influence on the phylogenetic reconstructions.

190 Consequently, all results presented from this point reflect those from the non-truncated $C O X 1$

191 alignment.

\section{Species delineation based on molecular data}

193 We implemented two DNA taxonomy approaches to evaluate the presence of cryptic species.

194 First, the general mixed Yule coalescent (GMYC) approach (Fujisawa \& Barraclough 2013;

195 Pons et al. 2006) was applied to an ultrametric tree (produced using BEAST) in R v2.15.3 (R

196 Development Core Team 2008) with the Splits package (http://splits.r-forge.r-project.org). The

197 GMYC model is a process-based approach that detects the threshold in a gene tree at which

198 within-species processes (i.e. coalescence) shift to between-species processes (i.e. speciation and

199 extinction). Second, we combined the Poisson Tree Processes model for species delimitation

200 (PTP) and a Bayesian implementation of PTP (bPTP) to infer putative species boundaries on a

201 given phylogenetic input tree (Zhang et al. 2013). The PTP/bPTP model, unlike the GMYC

202 model, requires a bifurcated phylogenetic tree rather than an ultrametric tree. PTP/dPTP models

203 speciation or branching events in terms of the number of substitutions. The following parameters

204 were used: MCMC, 500,000 generations; thinning, 100; burn-in, 0.1; seed, 123, and assessed

205 convergence in each case to ensure the reliability of the results. 


\section{Delineation based on the analyses of morphological measurements}

207 Proportions of several characters that approximate body shape were assessed in 39 female adult

208 specimens that were keyed out as U. yaschenkoi (according to Koch, 1977) and were collected at

20926 locations (Table 1, Fig1). Gender was determined by examining the genital opercula of adult

210 scorpions, with males having a small finger-like projection known as the genital papilla. Because

211 our collection contained only three males, the analyses were done only with females.

212 The following traits were measured under a microscope using an ocular ruler with 1-mm

213 precision: carapace length (CL), metasoma segment V length (MVL), telson length (SL),

214 pedipalp length (PL), chela length (ChL), pecten length (PecL) and pecten width (PecW). Ratios

215 of traits (e.g. CL/MVL, SL/PL etc.) gave in total 21 variables scored in each individual

216 (Supplemental file 4). These variables were treated as predictors in the Linear Discriminant

217 Analysis (LDA) implemented in the R package "MASS" (Venables \& Ripley 2002). LDA was

218 used to test whether the linear combination of 21 variables (ratios of morphological

219 measurements) can predict individual's membership to a mitochondrial lineage (putative

220 species). Strong predictive power of morphological variation on the observed molecular

221 divergence would provide additional support for a species complex in U. yaschenkoi.

\section{Divergence time estimation}

223 The mitochondrial gene tree was time calibrated with divergence times of nodes inferred from

$22495 \%$ highest posterior density (HPD) intervals. Scorpion-specific mutation rates of 0.007

225 substitutions/site/million years for $C O X I$ and 0.005 substitutions/site/million years for $16 S$

226 (Gantenbein et al. 2005a; Gantenbein \& Largiadèr 2003) were used to calibrate the tree. These

227 estimates are derived from buthid scorpions and have been used to estimate divergence times

228 among various scorpion lineages including non-buthid taxa (Bryson et al. 2013a; Bryson et al. 
229 2013b; Graham et al. 2012). Substitution rates were set in BEAUti v1.7.3 (Drummond et al.

230 2012) using relaxed clock log normal priors. Tracer was then used to obtain parameter estimates

231 for time to most recent common ancestor (tMRCAs) for nodes within the gene tree.

\section{Results}

234 We identified 31 unique mitochondrial haplotypes with uncorrected distances between

235 haplotypes ranging from $0.3-7.6 \%$ (mean \pm standard deviation $=3.0 \% \pm 0.4 \%$ ) and distances

236 from the outgroup taxa of $8.4-10.2 \%$ (mean \pm standard deviation $=9.4 \% \pm 1.4 \%)$ (Supplemental

237 File 1). A total of 13 nuclear $28 S$ haplotypes were identified with uncorrected $p$-distances of $0.1-$

$2380.5 \%($ mean \pm standard deviation $=0.2 \% \pm 0.1 \%)($ Supplemental File 2$)$. A list of haplotypes for

239 sample locations is provided in Supplemental File 3.

\section{Phylogenetic analysis}

241 Mitochondrial markers

242 Bayesian inference analysis of the mitochondrial dataset identified several genetically divergent

243 lineages (three major lineages represented as black, red and green clades in Fig2), with strong

244 statistical support for their respective monophyly (posterior probability $>0.95$ ). Sublineages

245 within the black clade are broadly distributed across Victoria, South Australia and Western

246 Australia, whereas the red and green clades are restricted to Western Australia (Fig1). From this

247 point forward we will refer to the black, red and green clades as the south-central (SC), western

248 (W) and central-western (CW) lineages, respectively. 
249 Mean uncorrected pairwise genetic distances between the three major lineages (SC, CW and W)

250 ranged from 6.4 to $6.9 \%$ (overall mean \pm standard deviation $=6.6 \% \pm 0.9 \%$ ). The mean sub-

251 lineage distances ranged from $2.2 \% \pm 0.4 \%$ and $0.8 \% \pm 0.2 \%$, respectively (not calculated for the

252 W lineage due to only a single recorded haplotype). Mean uncorrected distances between the

253 three major lineages and the outgroups ranged from 9.3 to $10.3 \%$ (mean \pm standard deviation $=$

$2549.4 \% \pm 1.4 \%)$

255 Nuclear marker

256 Despite low level of variation in the $28 S$ dataset, Bayesian analysis produced a nuclear gene

257 topology that was largely concordant with the mitochondrial gene tree. Three genetically

258 divergent clades were identified, corresponding to those from the mitochondrial dataset (SC, CW

259 and W, Fig3). In each case, strong statistical support for the monophyly of each clade was found

260 (posterior probability $>0.95$ ). The unresolved interrelationships among lineages within each

261 clade in the nuclear gene tree prevented any reliable inferences of phylogeographic patterns.

\section{Molecular-based species delineation}

263 Among the 31 unique mitochondrial haplotypes described above, the GMYC model identified

264 nine entities and the PTP/bPTP approach identified seven, each representing putative species

265 (Table 3). The assignment of haplotypes to putative species groups is shown in Fig2, where

266 conspecifics share a common number. Species assignments were highly consistent when

267 comparing each of the methods, but we presented the PTP/bPTP results as they are more

268 accurate when the evolutionary distances between lineages are small (Zhang et al. 2013). In

269 summary, SC, W and CW clades were recognized as putative species groups, as were the sub-

270 lineages within the SC ancestral grouping (SC-1 to 5, Fig2). 


\section{Discriminant power of morphological variation}

272 None of the $U$. yaschenkoi specimens that were characterized at 21 morphological ratio variables

273 were assigned to the $\mathrm{W}$ mitochondrial clade, hence the LDA was done on 39 females assigned to

274 the SC and the CW clades. Individuals were categorized into four groups (putative species)

275 based on the results of the PTP/bPTP molecular species delineation analysis: 18 females from

276 SC-1, 12 from SC-3, three from the SC-4, and six from the CW clade (Fig2). Because our dataset

277 contained four groups, we could find a maximum of three discriminant functions that separate

278 these groups.

279 The first discriminant function (LD1) achieved 93.7\% of the separation, reflecting the

280 morphological distinction of the CW clade from the SC clade (Fig4). Further separation of the

281 three putative groups within the SC clade was weak (LD2-3, Fig4). We then grouped samples

282 into two putative species (CW and SC clade) and tested the accuracy of prediction using 100

283 jackknife resampling steps. The grouping into two molecular clades based on morphological

284 variation was $100 \%$ accurate $(33 / 33)$ for the SC clade and $83.3 \%$ accurate $(5 / 6)$ for the $\mathrm{CW}$

285 clade. Therefore, our results indicate strong predictive power of body proportion variation on the

286 observed molecular divergence, and suggest the existence of at least two distinct taxa within $U$.

287 yaschenkoi.

288 The most discriminating uncorrelated proportions were of the telson and chela length (SL/ChL)

289 and pedipalp and pecten length (PL/PecL). Overall, members of the CW clade tend to have

290 disproportionately shortened chela and enlarged pecten when compared to the members of the

291 SC clade. 


\section{Divergence dating}

293 Our time calibrated mitochondrial phylogeny suggested that the split between the major $U$.

294 yaschenkoi clades (SC, CW and W lineages) occurred during the late Miocene/early Pliocene (4-

2957 MYA) (Fig2). Lineage diversifications within SC appear to have occurred during the Pliocene

296 and early Pleistocene (1.8-4 MYA), while finer-scale phylogeographic patterning within the sub-

297 lineages arose during the late Pleistocene (<1 Mya). Divergence time estimates should be

298 interpreted with some caution, as the nucleotide substitution rate was derived from a different

299 scorpion family (Buthidae) and there are large errors margins around 95\% HPD estimates.

300 Biogeographic patterns

301 The SC lineage showed substantial geographic structure. The most divergent sub-lineage (SC-5)

302 was found in Western Australia in sympatry with the CW lineage (Fig1). SC-1 was found west

303 of the Central Ranges, through to the Eyre Peninsula in South Australia, while SC-3 had a

304 distribution extending from the Central to Mt Lofty Ranges in South Australia, and across to

305 north-western Victoria. SC-4 had a narrow north-south distribution in the central inland and

306 coastal regions of South Australia (Fig1).

\section{Discussion}

309 Our analyses reveal strong genetic and morphological diversification in $U$. yaschenkoi across its

310 range, pointing to the existence of a species complex with at least three putative species High

311 statistical support for the monophyly and the extent of genetic divergence between the main three

312 lineages (6.4-6.9\%) exceeds estimates of interspecific divergence previously reported for other

313 scorpion and arthropod groups (Bryson et al. 2014; Tourinho et al. 2012; Wysocka et al. 2011). 
314 DNA-based species delineation approaches (GMYC and bPTP) provided significant statistical

315 support for the recognition of the three lineages ( $\mathrm{SC}, \mathrm{CW}, \mathrm{W})$ as distinct species, and potential

316 further cryptic speciation within the south-central clade (SC1-5, Fig2).

317 We also demonstrated a strong association between this molecular divergence and morphological

318 variation. Namely, ratios of several traits that approximate body shape had a strong predictive

319 power (83-100\%) in discriminating two major molecular clades (CW and SC). The two clades

320 differ most notably in proportions involving chela and pecten. Because of their great variation in

321 shape, scorpion chalae have been used as one of the key characters to delineate different

322 ecomorphotypes (van der Meijden et al. 2012). Until now $U$. yaschenkoi has been distinguished

323 from other congeneric species by its much smaller terminal prolateral tarsal ungues and by the

324 production of large amounts of venom (Koch 1977). Based on our results from a limited sample

325 size, detailed analyses of morphological variation in U. yaschekoi are warranted.

326 Our time-calibrated phylogeny suggests that the split between the $\mathrm{CW}, \mathrm{W}$ and $\mathrm{SC}$ clades

327 occurred during the mid-Miocene to early Pliocene (approximately 5-9 Mya). This geological

328 time was marked by a shift to a much drier climate, the significant contraction of rainforests and

329 the expansion of arid habitats (Martin 2006). Further diversification within the major ancestral

330 U. yaschenkoi lineages appears to have occurred throughout the Pliocene (3-5 Mya), which was a

331 consistently dry period. This is followed by further lineage divergence during the mid and late

332 Pleistocene when the climate was highly dynamic ( $<1$ Mya), with wetter and drier episodes

333 corresponding to interglacial and glacial cycles (McLaren \& Wallace 2010).

334 The spatio-temporal dynamics of diversification observed in U. yaschenkoi parallels those

335 reported in other Australian arid biota. Reviewing tens of dated phylogenies of the south-western 
336 Australian terrestrial fauna, including arthropods like crayfish and spiders, (Rix et al. 2015)

337 found a compelling commonality in the basal east-west lineage diversification during the first

338 half Miocene (until 10 Mya). The more xeric taxa currently occupying semi-arid and arid zones

339 seemed to have experienced this divergence in late Miocene (6-10 Mya) (Rix et al. 2015), which

340 we also inferred in the desert scorpion U. yaschenkoi (Fig2). A strong genetic and morphological

341 divergence between the $U$. yaschenkoi lineages from the western $(\mathrm{CW}, \mathrm{W})$ and south-central

342 (SC) Australia could be partly explained by the Miocene east-west vicariance hypothesis (Rix et

343 al. 2015) (Fig1). After a longer period of range contraction, arid-adapted taxa such as $U$.

344 yaschenkoi likely underwent significant range expansions during the Pliocene. Separation of SC-

3455 from other SC sub-lineages was estimated to have occurred during this time (Fig2), with SC-5

346 moving easterly. This sub-lineage is now sympatric with the CW clade (Fig1), suggesting their

347 secondary contact. Further diversification within the SC clade (SC1-4) coincides with transition

348 to the Pleistocene severe glacial cycles and expansion of the Australian deserts during the last 1

349 My (beginning of the "dusty world", (Rix et al. 2015)). Like the Bynoe's gecko (Fujita et al.

350 2010) and lizards (Dubey \& Shine 2010; Pepper et al. 2011), U. yaschenkoi is another arid-

351 adapted Australian taxon whose diversification and distribution were profoundly affected by the

352 opening of desert biomes during this hyper-arid, unstable climatic history. Teasing out the

353 relative importance of vicariance, putative refugia (e.g. Pilbara, Kimberley, central Ranges,

354 (Pepper et al. 2013)), or dispersal (Melville et al. 2016) (Fig1) in shaping this diversity would

355 require extensive sampling, particularly at the western and northern parts of $U$. yaschenkoi 356 distribution. 


\section{Revising the $U$. yaschenkoi taxonomy - future directions}

358 Our results provide solid baseline data on the historical and spatial extent of diversification in $U$.

359 yaschenkoi and offer some guidelines for future integrative taxonomic approaches in delimiting

360 species within this taxon. We found an agreement among disciplines (morphology, nuclear and

361 mitochondrial genetic information) during a primary exploration, which strengthens the

362 argument for a taxonomic revision (Pante et al. 2014; Schlick-Steiner et al. 2009). Congruent

363 morphological and molecular phylogenetic signals are particularly compelling for a scorpion

364 taxon, given that this is not the case in many scorpion lineages (Sharma et al. 2015).

365 The level of mitochondrial sequence divergence observed between $U$. yaschenkoi lineages satisfy

366 the requirements for species delineation based on the principles of the phylogenetic species

367 concept (De Queiroz 2007; Wheeler 1999), The three major lineages (SC, CW, W) can be

368 considered the putative species. Because genetic 'yardstick' approaches provide crude taxonomic

369 measures and nucleotide substitution rates often vary considerably between taxonomic groups,

370 some caution is needed when considering findings of these analyses alone. Additional DNA-

371 based species delineation approaches (GMYC and bPTP) indicated extensive cryptic speciation

372 in U. yaschenkoi (Fig. 2). The GMYC method has been criticized for over-splitting species with

373 a pronounced genetic structure (Satler et al. 2013), yet several recent studies have shown that it is

374 highly robust (Fujisawa \& Barraclough 2013; Talavera et al. 2013). The obvious next step is to

375 characterize the nuclear genome-wide variation in $U$. yaschenkoi sampled extensively within the

376 “type" locality $\left(28^{\circ} 35^{\prime} \mathrm{S}, 138^{\circ} 33^{\prime} \mathrm{E}\right)$, as well as western and northern parts of the distribution. We

377 certainly advise against a pool-sequencing phylogenomic approach (e.g. samples from the same

378 location are pooled to achieve cost-efficiency), given that the putative species have been found in 379 sympatry. 
380 The proportions of various morphological characters are routinely used in species descriptions or

381 identification keys, particularly for arthropods where morphologically similar species often differ

382 significantly in body proportions but not in qualitative characters.(Baur \& Leuenberger 2011).

383 Arguably, the results of multivariate analyses summarizing the overall body shape differences

384 between groups are not easily interpreted. Yet, our initial results suggest that further analyses of

385 e.g. chela shape might reveal more easily quantifiable diagnostic characters for U. yaschenkoi.

386 Several parameters of chala shape were found to be correlated with the amount of strain stress

387 they can withstand. Specifically, slender chela morphologies may be less suitable for high-force

388 functions such as burrowing and defence (van der Meijden et al. 2012). Given that U. yaschekoi

389 putative species (SC and $\mathrm{CW}$ ) show marked shape differences involving chela, further

390 exploration of burrowing behavior or pray preference might provide additional characters to

391 describe the $U$. yaschenkoi species complex.

392 Finally, it is important to note that we cannot exclude the possibility that some of the cryptic

393 lineages have already been described as species, and we are not able to compare our genetic data

394 against other Urodacus sequences as none published at the time of our study. Also, our sampling

395 did not cover the exact "type" locality $\left(28^{\circ} 35^{\prime} \mathrm{S}, 138^{\circ} 33^{\prime} \mathrm{E}\right)$. The samples closest to this area

396 belong to the SC clade and likely represent the "type" lineage. These data gaps would need to be

397 addressed in further studies aiming to revise the taxonomy of the Australian desert scorpion $U$.

398 yaschenkoi.

\section{Conclusions}

400 Our study provides the first insight into the molecular phylogeny of the endemic Australian

401 scorpion Urodacus yaschenkoi. We show that this scorpion shares a complex diversification 
402 history with other Australian arid-adapted fauna. Concordance between the mitochondrial and 403 nuclear data, along with the morphological variation, all suggest that $U$. yaschenkoi is a species 404 complex that requires further taxonomic revision. Our findings highlight the importance of 405 conserving populations from different Australian arid zones in order to preserve patterns of 406 endemism and evolutionary potential. 
408

409

410

411

412

413

414

415

416

417

418

419

420

421

422

423

424

425

426

427

428

429

430

431

432

433

434

435

436

437

438

439

440

441

442

443

444

445

446

447

448

449

450

451

\section{References}

Akaike H, and Company N-hP. 1981. Likelihood of a model and information criteria. Journal of Econometrics 16:3-14. 10.1016/0304-4076(81)90071-3

Arabi J, Judson MLI, Deharveng L, Lourenço WR, Cruaud C, and Hassanin A. 2012. Nucleotide composition of CO1 sequences in Chelicerata (arthropoda): Detecting new mitogenomic rearrangements. Journal of Molecular Evolution 74:81-95. 10.1007/s00239-012-9490-7

Baur H, and Leuenberger C. 2011. Analysis of Ratios in Multivariate Morphometry. Systematic Biology. 10.1093/sysbio/syr061

Birula A. 1903. Sur un nouveau genre et une nouvelle espèce de scorpions, provenant d'Australie.]. Exploration du Parc National de l'Upemba. Mission G F de Witte 8:xxxiiixxxiv.

Bouckaert R, Heled J, Kühnert D, Vaughan T, Wu CH, Xie D, Suchard MA, Rambaut A, and Drummond AJ. 2014. BEAST 2: A Software Platform for Bayesian Evolutionary Analysis. PLoS Computational Biology 10:e1003537. 10.1371/journal.pcbi.1003537

Bryson RW, Prendini L, Savary WE, and Pearman PB. 2014. Caves as microrefugia: Pleistocene phylogeography of the troglophilic North American scorpion Pseudouroctonus reddelli. BMC Evolutionary Biology 14:9. 10.1186/1471-2148-14-9

Bryson RW, Riddle BR, Graham MR, Smith BT, and Prendini L. 2013a. As Old as the Hills: Montane Scorpions in Southwestern North America Reveal Ancient Associations between Biotic Diversification and Landscape History. PLoS ONE 8:e52822. 10.1371/journal.pone.0052822

Bryson RW, Savary WE, Prendini L, and Parmakelis A. 2013b. Biogeography of scorpions in the Pseudouroctonus minimus complex (Vaejovidae) from south-western North America: Implications of ecological specialization for pre-Quaternary diversification. Journal of Biogeography 40:1850-1860. 10.1111/jbi.12134

De Queiroz K. 2007. Species concepts and species delimitation. Systematic Biology 56:879-886. $10.1080 / 10635150701701083$

Drummond A, and Rambaut A. 2007. Tracer: MCMC trace analysis tool. 1.5.0, Program distributed by the authors.

Drummond AJ, Suchard MA, Xie D, and Rambaut A. 2012. Bayesian phylogenetics with BEAUti and the BEAST 1.7. Molecular Biology and Evolution 29:1969-1973. 10.1093/molbev/mss075

Dubey S, and Shine R. 2010. Evolutionary Diversification of the Lizard Genus $<$ italic $>$ Bassiana</italic $>$ (Scincidae) across Southern Australia. PLoS ONE 5:e12982. 10.1371/journal.pone.0012982

Dunlop JA. 2010. Geological history and phylogeny of Chelicerata. Arthropod structure \& development 39:124-142.

Farris JS, Källersjö M, Kluge AG, and Bult C. 1995. Constructing a Significance Test for Incongruence. Systematic Biology 44:570-572. 10.1093/sysbio/44.4.570

Fet V, Polis GA, and Sissom WD. 1998. Life in sandy deserts: the scorpion model. p 609-622.

Fujisawa T, and Barraclough TG. 2013. Delimiting species using single-locus data and the generalized mixed yule coalescent approach: A revised method and evaluation on simulated data sets. Systematic Biology 62:707-724. 10.1093/sysbio/syt033 
452

453

454

455

456

457

458

459

460

461

462

463

464

465

466

467

468

469

470

471

472

473

474

475

476

477

478

479

480

481

482

483

484

485

486

487

488

489

490

491

492

493

494

495
Fujita MK, McGuire JA, Donnellan SC, and Moritz C. 2010. Diversification and persistence at the arid-monsoonal interface: australia-wide biogeography of the Bynoe's gecko (Heteronotia binoei; Gekkonidae). Evolution 64:2293-2314. 10.1111/j.15585646.2010.00993.x

Gantenbein B, Fet V, Gantenbein-Ritter IA, and Balloux F. 2005a. Evidence for recombination in scorpion mitochondrial DNA (Scorpiones: Buthidae). Proceedings of the Royal Society B: Biological Sciences 272:697-704. 10.1098/rspb.2004.3017

Gantenbein B, Fet V, Gantenbein-Ritter IA, and Balloux F. 2005b. Evidence for recombination in scorpion mitochondrial DNA (Scorpiones: Buthidae). Proceedings Biological sciences / The Royal Society 272:697-704. 10.1098/rspb.2004.3017

Gantenbein B, and Largiadèr CR. 2003. The phylogeographic importance of the Strait of Gibraltar as a gene flow barrier in terrestrial arthropods: A case study with the scorpion Buthus occitanus as model organism. Molecular Phylogenetics and Evolution 28:119130. 10.1016/S1055-7903(03)00031-9

Glauert L. 1963. Notes on Urodacus scorpions. . Western Australian Naturalist 8:132-135.

Graham MRMR, Oláh-Hemmings V, and Fet V. 2012. Phylogeography of co-distributed dune scorpions identifies the Amu Darya River as a long-standing component of Central Asian biogeography: (Scorpiones: Buthidae. Zoology in the Middle East 55:95-110. 10.1080/09397140.2012.10648924

Gurevitz M, Karbat I, Cohen L, Ilan N, Kahn R, Turkov M, Stankiewicz M, Stühmer W, Dong K, and Gordon D. 2007. The insecticidal potential of scorpion $\beta$-toxins. p 473-489.

Jeram AJ. 1997. Phylogeny, classification and evolution of Silurian and Devonian scorpions. Proceedings of the 17th European colloquium of arachnology, Edinburgh. p 17-31.

Kjellesvig-Waering EN. 1986. A restudy of the fossil Scorpionida of the world: Paleontological Research Institution.

Koch LE. 1977. The taxonomy, geographic distribution and evolutionary radiation of AustraloPapuan scorpions. Records of the Western Australian Museum 5:79-79.

Kraepelin K. 1916. Results of Dr. E. Mjöbergs Swedish Scientific Expeditions to Australia 19101913. 4. Scolopendriden und Scorpione. Arkiv för Zoologi 10:1-43.

Lourenço WR. 2001. The scorpion families and their geographical distribution.

Lourenço WR, and Cuellar O. 1995. Scorpions, scorpionism, life history strategies and parthenogenesis.

Luna-Ramírez K, Quintero-Hernández V, Vargas-Jaimes L, Batista CVF, Winkel KD, and Possani LD. 2013. Characterization of the venom from the Australian scorpion Urodacus yaschenkoi: Molecular mass analysis of components, cDNA sequences and peptides with antimicrobial activity. Toxicon 63:44-54. 10.1016/j.toxicon.2012.11.017

Martin HA. 2006. Cenozoic climatic change and the development of the arid vegetation in Australia. Journal of Arid Environments 66:533-563. 10.1016/j.jaridenv.2006.01.009

McLaren S, and Wallace MW. 2010. Plio-Pleistocene climate change and the onset of aridity in southeastern Australia. Global and Planetary Change 71:55-72. http://dx.doi.org/10.1016/j.gloplacha.2009.12.007

Melville J, Haines ML, Hale J, Chapple S, and Ritchie EG. 2016. Concordance in phylogeography and ecological niche modelling identify dispersal corridors for reptiles in arid Australia. Journal of Biogeography 43:1844-1855. 10.1111/jbi.12739 
496

497

498

499

500

501

502

503

504

505

506

507

508

509

510

511

512

513

514

515

516

517

518

519

520

521

522

523

524

525

526

527

528

529

530

531

532

533

534

535

536

537

538

539

540

Monod L, and Prendini L. 2015. Evidence for Eurogondwana: the roles of dispersal, extinction and vicariance in the evolution and biogeography of Indo-Pacific Hormuridae (Scorpiones: Scorpionoidea). Cladistics 31:71-111. 10.1111/cla.12067

Pante E, Schoelinck C, and Puillandre N. 2014. From Integrative Taxonomy to Species Description: One Step Beyond. Systematic Biology. 10.1093/sysbio/syu083

Pepper M, Doughty P, and Keogh JS. 2013. Geodiversity and endemism in the iconic Australian Pilbara region: a review of landscape evolution and biotic response in an ancient refugium. Journal of Biogeography 40:1225-1239. 10.1111/jbi.12080

Pepper M, Ho SYW, Fujita MK, and Scott Keogh J. 2011. The genetic legacy of aridification: Climate cycling fostered lizard diversification in Australian montane refugia and left lowlying deserts genetically depauperate. Molecular Phylogenetics and Evolution 61:750759. http://dx.doi.org/10.1016/j.ympev.2011.08.009

Pons J, Barraclough TG, Gomez-Zurita J, Cardoso A, Duran DP, Hazell S, Kamoun S, Sumlin WD, and Vogler AP. 2006. Sequence-based species delimitation for the DNA taxonomy of undescribed insects. Systematic Biology 55:595-609. 10.1080/10635150600852011

Posada D. 2008. jModelTest: Phylogenetic model averaging. Molecular Biology and Evolution 25:1253-1256. 10.1093/molbev/msn083

Possani LD, Merino E, Corona M, Bolivar F, and Becerril B. 2000. Peptides and genes coding for scorpion toxins that affect ion-channels. $\mathrm{p}$ 861-868.

Prendini L. 2010. Order Scorpiones C.L. Koch, 1837 scorpions. In: Gerlach J, and Marusik Y, eds. Arachnida and Myriapoda of the Seychelles islands. Manchester, UK: Siri Scientific Press, 321-330.

Prendini L, and Wheeler WC. 2005. Scorpion higher phylogeny and classification, taxonomic anarchy, and standards for peer review in online publishing. Cladistics 21:446-494. 10.1111/j.1096-0031.2005.00073.x

Rix MG, Edwards DL, Byrne M, Harvey MS, Joseph L, and Roberts JD. 2015. Biogeography and speciation of terrestrial fauna in the south-western Australian biodiversity hotspot. Biological Reviews 90:762-793. 10.1111/brv.12132

Rodríguez de la Vega RC, Schwartz EF, and Possani LD. 2010. Mining on scorpion venom biodiversity. p 1155-1161.

Satler JD, Carstens BC, and Hedin M. 2013. Multilocus species delimitation in a complex of morphologically conserved trapdoor spiders (mygalomorphae, antrodiaetidae, Aliatypus). Systematic Biology 62:805-823. 10.1093/sysbio/syt041

Schlick-Steiner BC, Steiner FM, Seifert B, Stauffer C, Christian E, and Crozier RH. 2009. Integrative Taxonomy: A Multisource Approach to Exploring Biodiversity. Annual Review of Entomology 55:421-438. 10.1146/annurev-ento-112408-085432

Sharma PP, Fernández R, Esposito LA, González-Santillán E, and Monod L. 2015. Phylogenomic resolution of scorpions reveals multilevel discordance with morphological phylogenetic signal. Proceedings of the Royal Society B: Biological Sciences 282.

Sharma PP, Kaluziak ST, Pérez-Porro AR, González VL, Hormiga G, Wheeler WC, and Giribet G. 2014. Phylogenomic interrogation of Arachnida reveals systemic conflicts in phylogenetic signal. Molecular Biology and Evolution.

Shultz JW. 2007. A phylogenetic analysis of the arachnid orders based on morphological characters. Zoological Journal of the Linnean Society 150:221-265. 10.1111/j.10963642.2007.00284.x 
541

542

543

544

545

546

547

548

549

550

551

552

553

554

555

556

557

558

559

560

561

562

563

564

565

566

567

568

569

570

571

572

573

574

575

576

577

578

579

580

581

582

583

584

585

586
Simon C, Frati F, Beckenbach A, Crespi B, Liu H, and Flook P. 1994. Evolution, weighting, and phylogenetic utility of mitochondrial gene sequences and a compilation of conserved PCR primers. Annals of the Entomological Society of America 87:651-701.

Soleglad ME, and Fet V. 2003. High-level systematics and phylogeny of the extant scorpions (Scorpiones: Orthosterni). . Euscorpius 11:1-175.

Stachel SJ, Stockwell SA, and Van Vranken DL. 1999. The fluorescence of scorpions and cataractogenesis. Chemistry and Biology 6:531-539. 10.1016/S1074-5521(99)80085-4

Swofford DL. 2002. PAUP* phylogenetic analysis using parsimony (*and other methods). Version 4.0b10. Sinauer Associates. 10.1159/000170955

Talavera G, Dincă V, Vila R, and Paradis E. 2013. Factors affecting species delimitations with the GMYC model: insights from a butterfly survey. Methods in Ecology and Evolution 4:1101-1110. 10.1111/2041-210x.12107

Tanaka H, Roubik DW, Kato M, Liew F, and Gunsalam G. 2001. Phylogenetic position of Apis nuluensis of northern Borneo and phylogeography of A. cerana as inferred from mitochondrial DNA sequences. p 44-51.

Tourinho JL, Sole-Cava AM, and Lazoski C. 2012. Cryptic species within the commercially most important lobster in the tropical Atlantic, the spiny lobster Panulirus argus. Marine Biology 159:1897-1906. http://dx.doi.org/10.1007/s00227-012-1977-7

van der Meijden A, Kleinteich T, and Coelho P. 2012. Packing a pinch: functional implications of chela shapes in scorpions using finite element analysis. Journal of Anatomy 220:423434. 10.1111/j.1469-7580.2012.01485.x

Venables W, and Ripley B. 2002. Modern Applied Statistics with S. Fourth Edition ed. New York: Springer.

Volschenk ES, Harvey MS, and Prendini L. 2012. A new species of Urodacus (Scorpiones: Urodacidae) from Western Australia. American Museum Novitiates 3748:1-18. 10.1206/3748.2

Volschenk ES, Mattoni CI, and Prendini L. 2008. Comparative anatomy of the mesosomal organs of scorpions (Chelicerata, Scorpiones), with implications for the phylogeny of the order. p 651-675.

Volschenk ES, and Prendini L. 2008. Aops oncodactylus, gen. et sp. nov., the first troglobitic urodacid (Urodacidae:Scorpiones), with a re-assessment of cavernicolous, troglobitic and troglomorphic scorpions. Invertebrate Systematics 22:235-257. 10.1071/IS06054

Walker, K.L, Yen AL, and Milledge, G.A. . 2003. . Spiders and Scorpions commonly found in Victoria. . Melbourne, Australia: The Royal Society of Victoria.

Wheeler QD. 1999. Why the phylogenetic species concept?-Elementary. Journal of nematology 31:134-141.

Wysocka A, Krzysztofiak L, Krzysztofiak A, Zołnierkiewicz O, Ojdowska E, and Sell J. 2011. Low genetic diversity in Polish populations of sibling ant species: Lasius niger (L.) and Lasius platythorax Seifert (Hymenoptera, Formicidae). Insectes Sociaux 58:191-195. 10.1007/s00040-010-0135-9

Xu X, Duan Z, Di Z, He Y, Li J, Li Z, Xie C, Zeng X, Cao Z, Wu Y, Liang S, and Li W. 2014. Proteomic analysis of the venom from the scorpion Mesobuthus martensii. Journal of Proteomics 106:162-180. http://dx.doi.org/10.1016/j.jprot.2014.04.032

Zhang J, Kapli P, Pavlidis P, and Stamatakis A. 2013. A general species delimitation method with applications to phylogenetic placements. Bioinformatics 29:2869-2876. 10.1093/bioinformatics/btt499 


\section{Web references:}

588 Department of the Environment, Water, Heritage and the Arts (12 February 2010). "Species 589 Urodacus yaschenkoi (Birula, 1903)".Australian Biological Resources Study: Australian Faunal 590 Directory. Commonwealth of Australia. Retrieved 20 July 2015.

591 CITES Appendix II, http://www.cites.org/eng/app/appendices.php; accessed on Sep 10, 2014.

592 Medscape, http://emedicine.medscape.com/article/168230-overview; accessed on Aug 20, 2014. 
593 Table 1. Urodacus yaschenkoi specimen location and analyses made. List of Urodacus

594 yaschenkoi collected from the field as live specimens (Field) or obtained from the Australian

595 museum collections (South Australian Museum - SA, Western Australian Museum - WA).

596 Geographic position (lat/log) and the geographic region details are reported for each sample. List

597 of haplotypes (mito, 28S) and GenBank Accession \# scored in each individual. Morphological

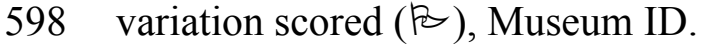

\begin{tabular}{|c|c|c|c|c|c|c|c|c|c|}
\hline Sample & Source & Latitude & Longitude & $\begin{array}{l}\text { Geographic } \\
\text { Region }\end{array}$ & $\begin{array}{l}\text { mito } \\
\text { Haplotype }\end{array}$ & $\begin{array}{l}28 \mathrm{~S} \\
\text { Haplotype }\end{array}$ & $\begin{array}{l}\text { GenBank } \\
\text { (mito / 28S) }\end{array}$ & Morpho & $\begin{array}{l}\text { Museum } \\
\text { ID/Reg.No. }\end{array}$ \\
\hline BKA11 & Field & -33.2283 & 141.3011 & NSW & 20 & 1 & $\begin{array}{l}\text { KP176775/ } \\
\text { KP176743 }\end{array}$ & & NA \\
\hline BKA12 & Field & -33.2283 & 141.3011 & NSW & 20 & 2 & $\begin{array}{l}\text { KP176775 / } \\
\text { KP176744 }\end{array}$ & & NA \\
\hline BKB08 & Field & -33.2199 & 141.3089 & NSW & 20 & 1 & & & NA \\
\hline BKB12 & Field & -33.2242 & 141.3061 & NSW & 20 & 1 & $\begin{array}{l}\text { KP176775 / } \\
\text { KP176743 }\end{array}$ & & NA \\
\hline BK13 & Field & -33.2283 & 141.3011 & NSW & 20 & 1 & & & NA \\
\hline MARR1 & Field & -26.3400 & 133.2000 & SA & 28 & 3 & $\begin{array}{l}\text { KP176783 / } \\
\text { KP176745 }\end{array}$ & & NA \\
\hline MARR2 & Field & -26.3400 & 133.2000 & SA & 28 & 3 & $\begin{array}{l}\text { KP176783 / } \\
\text { KP176745 }\end{array}$ & & NA \\
\hline PIM1 & Field & -31.2509 & 136.5089 & SA & 1 & 4 & $\begin{array}{l}\text { KP176756/ } \\
\text { KP176746 }\end{array}$ & & NA \\
\hline PIM2 & Field & -31.2509 & 136.5089 & SA & 1 & 5 & $\begin{array}{l}\text { KP176756/ } \\
\text { KP176747 }\end{array}$ & & NA \\
\hline PIM5 & Field & -31.2509 & 136.5089 & SA & 1 & 4 & $\begin{array}{l}\text { KP176756/ } \\
\text { KP176746 }\end{array}$ & & NA \\
\hline PIM6 & Field & -31.2509 & 136.5089 & SA & 1 & 1 & $\begin{array}{l}\text { KP176756/ } \\
\text { KP176743 }\end{array}$ & & NA \\
\hline PIM8 & Field & -31.2509 & 136.5089 & SA & 1 & 1 & $\begin{array}{l}\text { KP176756/ } \\
\text { KP176743 }\end{array}$ & & NA \\
\hline POP1 & Field & -33.0710 & 141.6372 & NSW & 20 & 1 & $\begin{array}{l}\text { KP176775 / } \\
\text { KP176743 }\end{array}$ & & NA \\
\hline POP4 & Field & -33.0710 & 141.6372 & NSW & 20 & - & KP176775 & & NA \\
\hline POP5 & Field & -33.0710 & 141.6372 & NSW & 20 & - & KP176775 & & NA \\
\hline
\end{tabular}




\begin{tabular}{|c|c|c|c|c|c|c|c|c|c|}
\hline SAM1397 & SAM & -30.7667 & 138.1767 & SA & 2 & - & KP176757 & B & NS1397 \\
\hline SAM1399 & SAM & -27.1192 & 132.8300 & SA & 6 & - & KP176761 & $p$ & NS1399 \\
\hline SAM1400 & SAM & -27.1191 & 132.8300 & SA & 6 & - & KP176761 & $\beta$ & NS1400 \\
\hline SAM1403 & SAM & -26.6453 & 132.8858 & SA & 4 & - & KP176759 & B & NS1403 \\
\hline SAM1406 & SAM & -31.2878 & 136.5831 & SA & 1 & - & KP176756 & B & NS1406 \\
\hline SAM1412 & SAM & -26.2747 & 137.3269 & SA & 20 & - & KP176775 & $\beta$ & NS1412 \\
\hline SAM1415 & SAM & -33.8555 & 140.5361 & SA & 20 & - & KP176775 & B & NS1415 \\
\hline SAM1416 & SAM & -34.0583 & 140.1500 & SA & 20 & - & KP176775 & ß & NS1416 \\
\hline SAM1606 & SAM & -26.6922 & 134.1722 & SA & 23 & - & KP176778 & & NS1606 \\
\hline SAM1607 & SAM & -26.5767 & 137.1933 & SA & 22 & - & KP176777 & & NS1607 \\
\hline SAM1812 & SAM & -33.3267 & 137.0931 & SA & 15 & - & KP176770 & B & NS1812 \\
\hline SAM1823 & SAM & -33.7511 & 140.2747 & SA & 20 & - & KP176775 & B & NS1823 \\
\hline SAM1825 & SAM & -33.7230 & 140.1238 & SA & 20 & - & KP176775 & $\beta$ & NS1825 \\
\hline SAM1831 & SAM & -33.7183 & 139.9300 & SA & 20 & - & KP176775 & ß & NS1831 \\
\hline SAM1834 & SAM & -33.7236 & 139.0438 & SA & 20 & - & KP176775 & B & NS1834 \\
\hline SAM1835 & SAM & -33.7236 & 139.0438 & SA & 21 & - & KP176776 & $\beta$ & NS1835 \\
\hline SAM1837 & SAM & -33.7400 & 139.0816 & SA & 20 & - & KP176775 & B & NS1837 \\
\hline SAM1917 & SAM & -32.6244 & 135.0322 & SA & 24 & - & KP176779 & 同 & NS1917 \\
\hline SAM1939 & SAM & -33.1233 & 136.0214 & SA & 3 & - & KP176758 & $\beta$ & NS1939 \\
\hline SAM2038 & SAM & -33.1167 & 136.0000 & SA & 3 & - & KP176758 & 同 & NS2038 \\
\hline SAM2053 & SAM & -24.4036 & 132.8886 & NT & 14 & - & KP176769 & 同 & NS2053 \\
\hline SAM2054 & SAM & -28.4627 & 129.0102 & SA & 5 & - & KP176760 & ß & NS2054 \\
\hline SAM2055 & SAM & -28.4627 & 129.0102 & SA & 5 & - & KP176770 & 同 & NS2055 \\
\hline SAM2056 & SAM & -28.4627 & 129.0102 & SA & 10 & - & KP176765 & 同 & NS2056 \\
\hline SAM2060 & SAM & -28.4977 & 129.3205 & SA & 11 & - & KP176766 & ß & NS2060 \\
\hline SAM2061 & SAM & -28.4977 & 129.3205 & SA & 11 & - & KP176766 & 巴 & NS2061 \\
\hline SAM2062 & SAM & -24.5060 & 129.2619 & NT & 9 & - & KP176764 & ß & NS2062 \\
\hline SAM2067 & SAM & -32.0033 & 135.6558 & SA & 3 & - & KP176758 & & NS2067 \\
\hline SAM2070 & SAM & -28.8969 & 132.7575 & SA & 12 & - & KP176767 & p & NS2070 \\
\hline SAM2071 & SAM & -28.8969 & 132.7575 & SA & 13 & - & KP176768 & B & NS2071 \\
\hline SAM2073 & SAM & -28.5319 & 131.6903 & SA & 19 & - & KP176774 & & NS2073 \\
\hline
\end{tabular}




\begin{tabular}{|c|c|c|c|c|c|c|c|c|c|}
\hline SAM2076 & SAM & -29.7706 & 131.1081 & SA & 18 & - & KP176773 & & NS2076 \\
\hline SAM2120 & SAM & -31.9972 & 140.0644 & SA & 20 & - & KP176775 & $B$ & NS2120 \\
\hline SAM2125 & SAM & -29.1286 & 135.6997 & SA & 25 & - & KP176780 & 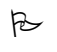 & NS2125 \\
\hline SAM2126 & SAM & -29.1286 & 135.6997 & SA & 20 & - & KP176775 & & NS2126 \\
\hline SAM2133 & SAM & -32.4947 & 135.3644 & SA & 7 & - & KP176762 & $\beta$ & NS2133 \\
\hline SAM2140 & SAM & -29.4053 & 132.8556 & SA & 26 & - & KP176781 & 12 & NS2140 \\
\hline WAM20 & WAM & -27.4867 & 122.3119 & WA & 31 & 7 & $\begin{array}{l}\text { KP176786/ } \\
\text { KP176749 }\end{array}$ & $B$ & 85020 \\
\hline WAM31 & WAM & -27.4867 & 122.3119 & WA & 31 & 8 & $\begin{array}{l}\text { KP176786 / } \\
\text { KP176750 }\end{array}$ & B & 85031 \\
\hline WAM32 & WAM & -27.4867 & 122.3119 & WA & 30 & 8 & $\begin{array}{l}\text { KP176785 / } \\
\text { KP176750 }\end{array}$ & B & 85032 \\
\hline WAM36 & WAM & -27.3893 & 115.1847 & WA & 29 & 9 & $\begin{array}{l}\text { KP176784 / } \\
\text { KP176751 }\end{array}$ & & 78236 \\
\hline WAM37 & WAM & -27.6145 & 121.9947 & WA & 17 & 10 & $\begin{array}{l}\text { KP176772 / } \\
\text { KP176752 }\end{array}$ & & 112637 \\
\hline WAM38 & WAM & -26.4408 & 115.3661 & WA & 29 & 9 & $\begin{array}{l}\text { KP176784 / } \\
\text { KP176751 }\end{array}$ & & 78238 \\
\hline WAM46 & WAM & -28.7333 & 123.8667 & WA & 16 & 11 & $\begin{array}{l}\text { KP176771/ } \\
\text { KP176753 }\end{array}$ & & 80246 \\
\hline WAM55 & WAM & -27.4867 & 122.3119 & WA & 31 & 7 & $\begin{array}{l}\text { KP176786/ } \\
\text { KP176749 }\end{array}$ & $\beta$ & 83855 \\
\hline WAM56 & WAM & -27.4867 & 122.3119 & WA & 30 & 7 & $\begin{array}{l}\text { KP176785 / } \\
\text { KP176749 }\end{array}$ & 屋 & 83856 \\
\hline WAM75 & WAM & -27.4867 & 122.3119 & WA & 31 & 12 & $\begin{array}{l}\text { KP176786 / } \\
\text { KP176754 }\end{array}$ & $\beta$ & 83875 \\
\hline WAM88 & WAM & -25.9307 & 128.4526 & WA & 8 & 13 & $\begin{array}{l}\text { KP176763/ } \\
\text { KP176755 }\end{array}$ & & 95988 \\
\hline Um1814 & SAM & -33.1997 & 138.2189 & SA & NA & NA & & & NS0001814 \\
\hline Um2714 & SAM & -33.1997 & 138.2189 & SA & NA & NA & & & NS0002714 \\
\hline Un2112 & SAM & -31.6597 & 129.1083 & SA & NA & NA & & & NS0002112 \\
\hline
\end{tabular}

599 NSW: New South Wales; SA: South Australia; WA: Western Australia; NT: Northern Territory

600 NA: Not applicable.

601

602 
603 Table 2. List of primer sequences and corresponding amplicons sizes for the three 604 Urodacus yaschenkoi loci (COXI, 16S rRNA, 28S rRNA).

\begin{tabular}{lllll}
\hline Marker & Primer & Primer sequence & Size (bp) & Reference \\
\hline COXI & F C1-J-2183 & 5'-CAACATTTATTTTGATTTTTTGG - 3' & $550-630$ & (Simon et al., 1994) \\
& R COXIKG-R2 & 5'-GATATTAATCCTAAAAAATGTTGAGG-3' & & (Tanaka et al., 2001) \\
COXI & Nested F & 5'-AGGAACCTTTTGGGGCTTT-3' & 150 & \\
COXI & Nested R & 5'-AGGAACCTTTTGGGGCTTT-3' & & \\
16S & F 16SF & 5'-AACAAAACCCACAGCTCACA-3' & 422 & (Gantenbein et al., \\
& R 16SR & 5'-GTGCAAAGGTAGCATAATCA-3' & & 2005) \\
28S & R1 & F R1S (5'-ACCCGCTGAATTTAAGCAT-3'), & 1158 & (Arabi et al., 2012) \\
& & R R1AS (5'-GCTATCCTGAGGGAAACTTC-3') & & \\
& R2 & F R2S (5'-CGACCCGTCTTGAAACACGGA-3'), & 1246 & \\
& R R2AS (5'-CACCTTGGAGACCTGCTGCGGAT-3') & & \\
\hline
\end{tabular}

\section{5}

606 
607 Table 3. Species delineation analyses in Urodacus yaschenkoi based on 31 unique 608 mitochondrial haplotypes.

\begin{tabular}{lcl}
\hline Analysis type & \# Entities & Statistics \\
\hline GMYC & 9 & Likelihood null model: $32.7519 ;$ likelihood best model: \\
& & $\begin{array}{l}33.36569 ; \text { likelihood ratio: } 1.2255 ; \text { P-value, } 0.0001, \\
\text { confidence interval: } 1-10\end{array}$ \\
& & Acceptance rate: $0.50975 ;$ merge: $49942 ;$ split: 50058 \\
PTP/bPTP (ML and BL) & 7 &
\end{tabular}

609

610 


\section{Supplemental Information}

612 The data sets supporting the results of this article are included within the article and its additional

613 files in Supplemental_Files_1-4.xlsx

614

615 Supplemental File 1. Pairwise uncorrected $p$-distance between 31 unique $U$. yaschenkoi

616 haplotypes and three outgroup haplotypes (U. novaehollandiae and two U. manicatus).

617 Haplotypes were generated from the concatenated partial sequences of COXI and 16S loci.

618 Supplemental File 2. Pairwise uncorrected $p$-distance between 13 unique $U$. yaschenkoi

619 haplotypes generated from the partial 28S sequence.

620 Supplemental File 3. List of haplotype numbers assigned to the U. yaschenkoi samples.

621 Supplemental File 4. Measures (in $\mathrm{mm}$ ) of seven morphological traits in U. yaschenkoi adult 622 females.

623

624 


\section{Figure captions}

626 Fig1. Urodacus yaschenkoi sampling locations across its distribution range (in dark yellow,

627 adapted from (Koch 1977)). Numbers 1 to 5 designate individuals belonging to the sub-lineages

$628(\mathrm{SC} 1-5)$ of the south-central major clade (SC); members of the central-western (CW) clade and

629 western (W) clades are marked in green and red color, respectively. Different hypotheses about

630 diversification in various Australian taxa (vicariance, refugia, dispersal corridors) are adapted

631 from (Melville et al. 2016; Pepper et al. 2011; Rix et al. 2015).

632

633 Fig2. Dated phylogeny (Bayesian tree) for Urodacus yaschenkoi based on the concatenated

634 COXI and $16 S$ partial sequences. Putative species inferred with the PTP/bPTP approach are

635 marked as SC1-5, CW and W. 95\% CI for each divergence time is shown in blue

636

637 Fig3. Bayesian unrooted tree for Urodacus yaschenkoi based on the $28 S$ partial sequences.

638

639 Fig4. LDA for body proportions. Individual scores for the first 3 axes of Linear Discriminant

640 Analysis. 21 body-proportions were measure in Urodacus yaschenkoi adult females. Numbers

$641(1,3,4)$ denote individuals belonging to one of the SC sublineages $(\mathrm{SC} 1,3,4)$, and $\mathrm{CW}$ denotes

642 individuals from the CW clade. 
643 Fig1.

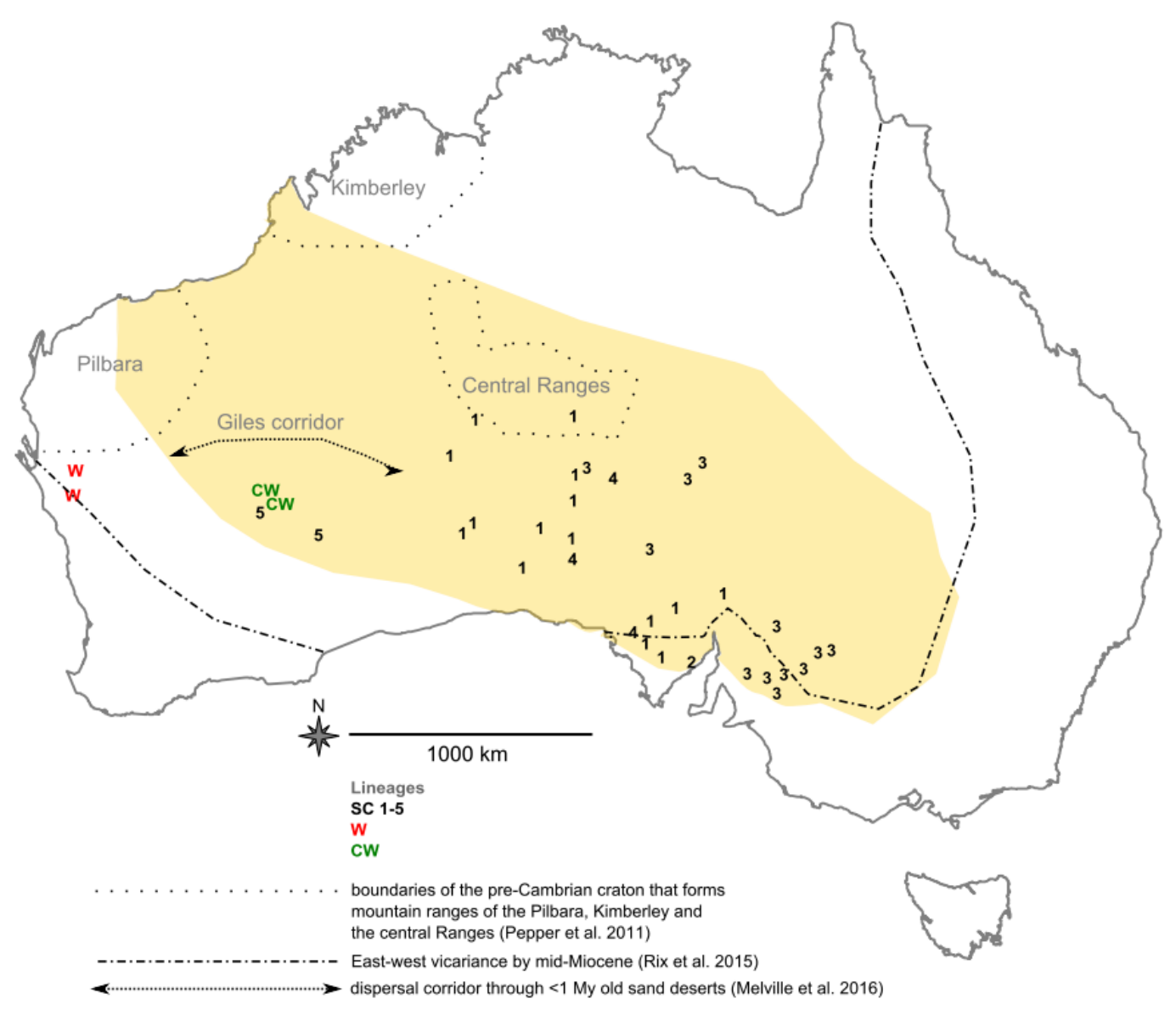


644 Fig2.

645

646

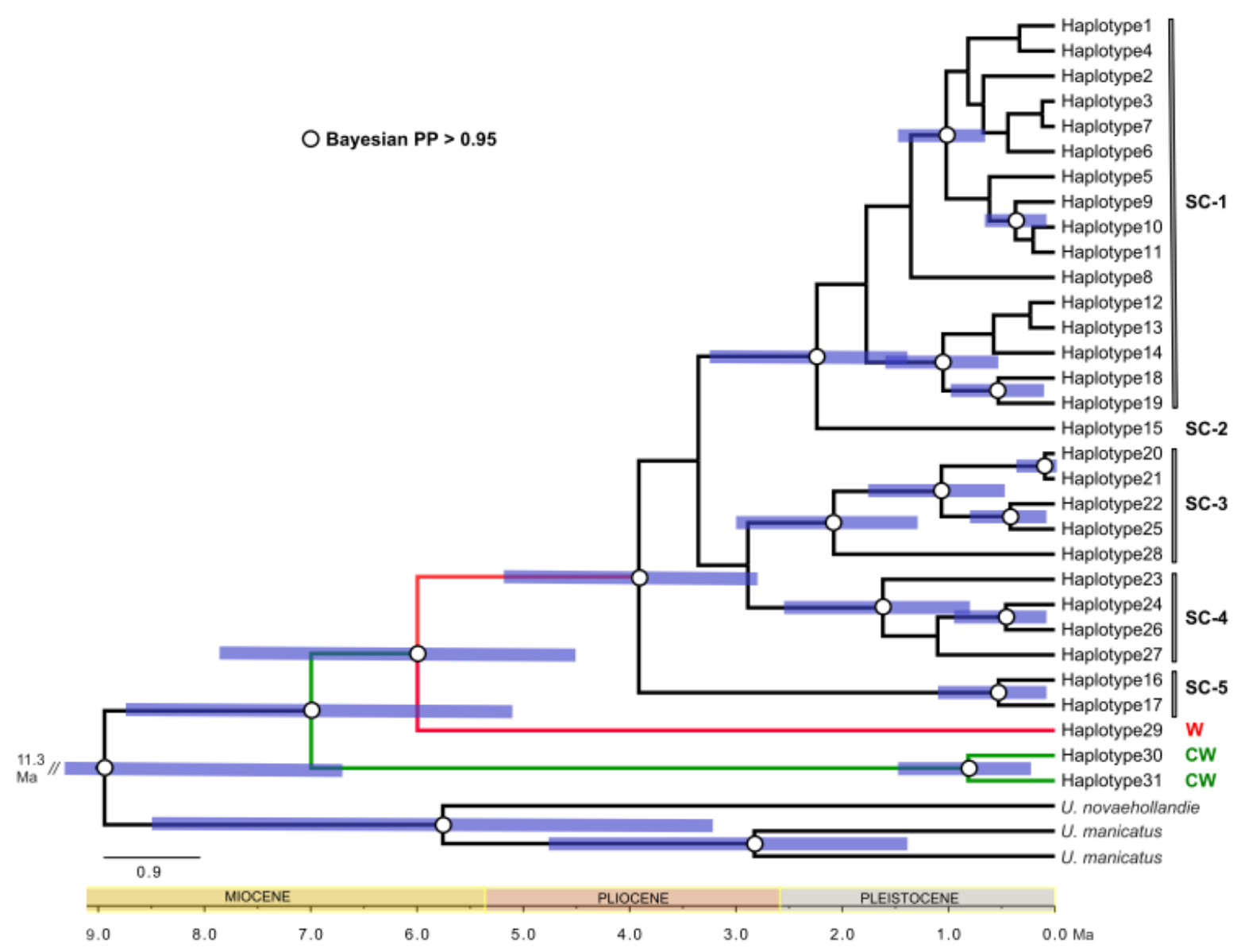


647 Fig3.

648

$649 \quad \mathrm{O}$ Bayesian PP $>0.95$
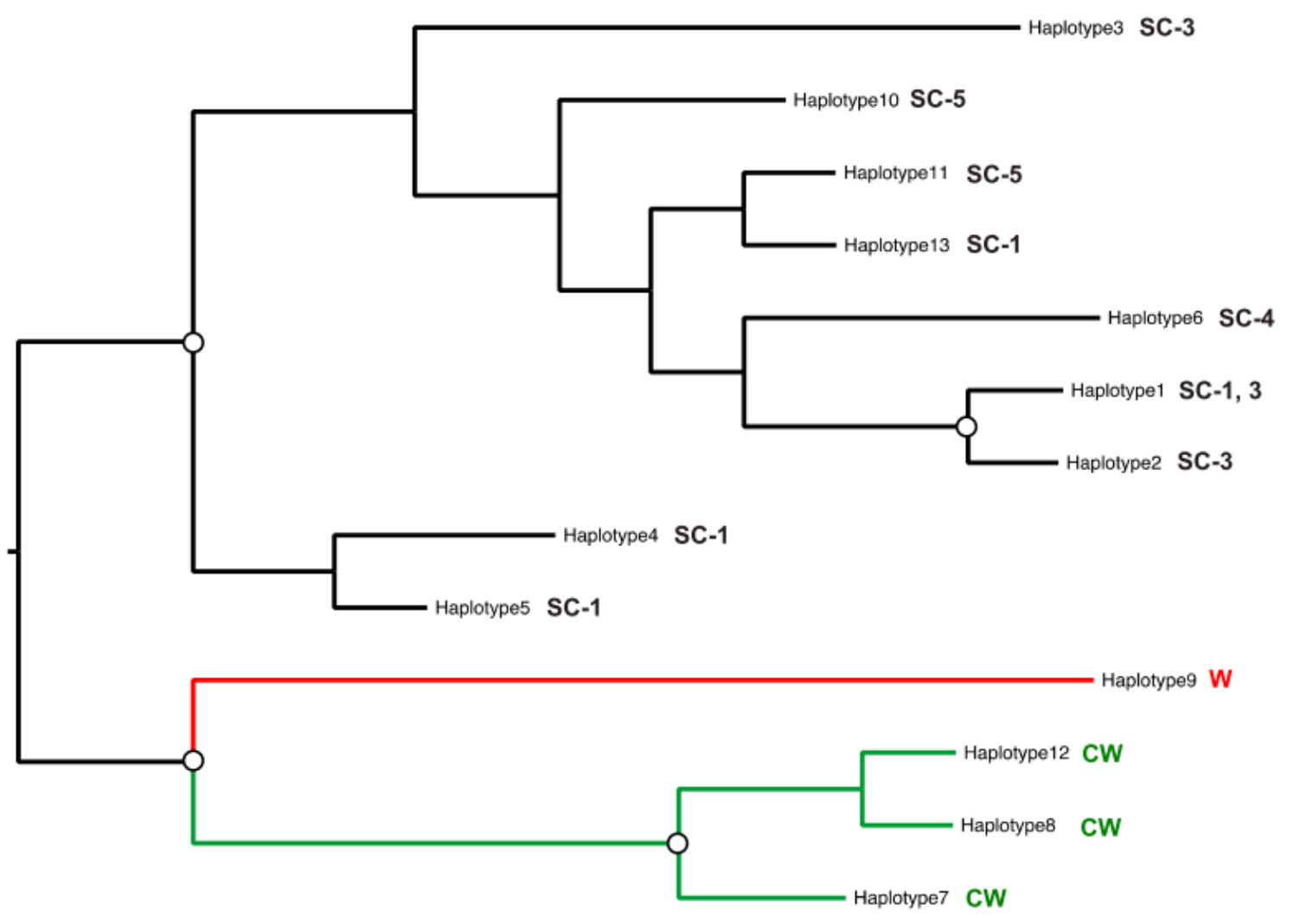

$2.0 \mathrm{E}-4$ 
$650 \quad$ Fig4.

651
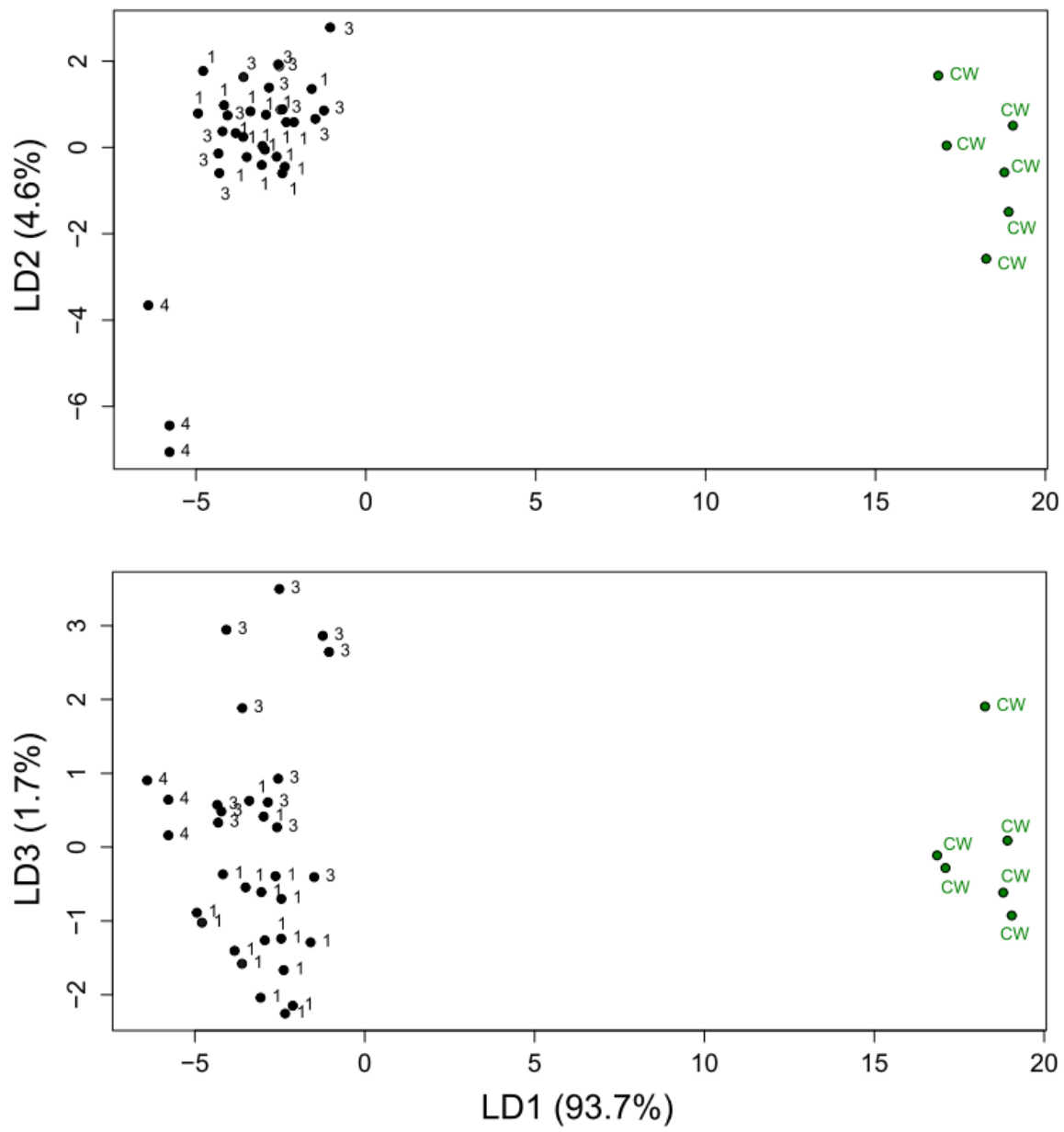